\title{
UNA MIRADA A LA POBLACIÓN DE BAJOS INGRESOS DEL ÁREA METROPOLITANA DE POSADAS DESDE LA MOVILIDAD COTIDIANA
}

Lic. Hector Daniel Blanco

Becario Doctoral. CONICET

Gabinete de Geografía. Facultad de Humanidades. Universidad Nacional de Formosa

E-mail: danielblancogeo@gmail.com

\section{RESUMEN}

A través del presente artículo se intenta proponer una visión analítica de los desplazamientos de la población de bajos ingresos económicos (indigentes y pobres) desde una dimensión material, es decir, según los viajes efectivamente realizados. Para ello, se han considerado indicadores que conciernen a la relación movilidad-ingreso y que conforman los datos secundarios de las Encuestas de Origen Destino, aplicada al Área Metropolitana de Posadas en el año 2010.

Se proporciona un breve panorama de la movilidad materializada, en un área que posee un $27,5 \%$ de hogares que se encuentran debajo de la línea de pobreza y de indigencia. No obstante, es menester reconocer que la comprensión de la movilidad desde un enfoque integral, no concluye en los viajes materializados, puesto que también incluye un universo subjetivo que requiere otras herramientas y definiciones metodologías adicionales para su correcto tratamiento.

\section{PALABRAS CLAVES}

Movilidad cotidiana; transporte; territorio; accesibilidad; pobreza.

\section{A LOOK AT THE LOW-INCOME POPULATION OF THE METROPOLITAN AREA OF POSADAS FROM DAILY MOBILITY}

\section{ABSTRACT}

Through this article attempts to propose an analytical view of the movement of the lowincome population (indigent and poor) in the territory of AMP, from a material dimension, that is, as the travel actually incurred. To do this, they have been considered indicators concerning mobility-income ratio and make the secondary data Origin Destination Surveys applied to the Metropolitan Area of Posadas in 2010.

A brief overview of mobility materialize, in an area that has a $27,5 \%$ of households are below the poverty line and poverty is provided. However, we must recognize that the understanding of mobility with a comprehensive approach not ends in travel materialized, since it also includes a subjective universe that requires additional definitions other tools and methodologies for proper treatment.

\section{KEY WORDS}

Daily mobility; transport; territory; accessibility; poverty.

Publicado en formato digital: Lic. Hector Daniel Blanco. UNA MIRADA A LA POBLACIÓN DE BAJOS INGRESOS DEL ÁREA METROPOLITANA DE POSADAS DESDE LA MOVILIDAD COTIDIANA. Revista Geográfica Digital. IGUNNE. Facultad de Humanidades. UNNE. Año 12. N No 24. Julio - Diciembre 2015. ISSN 1668-5180 Resistencia, Chaco.

En: http://hum.unne.edu.ar/revistas/geoweb/default.htm 


\section{INTRODUCCIÓN}

Al realizar un recorrido por la literatura especializada en el estudio del transporte, la movilidad y el territorio, diferentes autores manifiestan un consenso acerca de la transición o migración del foco de interés, yendo desde los medios de transportes hacia las personas y la movilidad cotidiana, o en otras palabras, desde de los medios materiales que permiten o son vectores del desplazamiento (la "oferta" de infraestructuras y servicios de transporte), hacia las personas que los usan y con el ello al plano subjetivo, dando lugar así a un cambio en el paradigma de pensamiento, que claramente pretende diferenciarse de las dimensiones euclidianas del desplazamiento.

Buena parte de las críticas a este modelo basado en la ingeniería del transporte, y a menudo simplemente en el aspecto cuantitativo de los desplazamientos dentro del sistema de transporte: números de viajes/día, pasajeros/km/día, tiempo medio del viaje, etc., (Lizarraga, 2012), se dirigen al supuesto básico de la racionalidad absoluta de las personas (Pattaroni et al., 2009, citado por Hernández et al., 2011 p. 3). Sin embargo, a partir de las significativas transformaciones urbanas de los años noventa, el concepto de movilidad comienza a ser revisado y enriquecido, incorporando las dimensiones subjetivas y contextuales del viaje. Al respecto, Gutiérrez a lo largo de una extensa labor investigativa sobre el tema (2006, 2009, 2012, etc.), realiza importantes aportaciones en pos de refundar las concepciones -tradicionales- de movilidad, de transporte, y de la propia unidad de estudio: el viaje. La autora sustenta que la movilidad es una práctica social de desplazamiento entre lugares con el fin de concretar actividades cotidianas. Involucra el desplazamiento de las personas y sus bienes, y conjuga deseos y/o necesidades de viaje (o requerimientos de movilidad) y capacidades objetivas y subjetivas de satisfacerlos, de cuya interacción resultan las condiciones de acceso de grupos sociales a bienes o servicios (la salud, la educación, etc.) y no a lugares físicos (el hospital, la escuela, etc.)

En esta misma línea de pensamiento, George Amar (2011), en su obra titulada Homo Movilis, afirma que la movilidad es entendida cada vez más en términos de creación de relaciones, de oportunidades y de sinergias, más que como un pasaje de distancias a una velocidad cada vez mayor. De hecho, la transición transporte-movilidad se inscribe en el marco de una evolución más profunda correspondiente a la "emergencia de una vida móvil" (y, por otra parte, de una ciudad móvil). Mientras que el paradigma clásico del transporte está centrado en la eficacia, la fiabilidad y la seguridad de la "gestión de flujo", concebida como flujo de partículas-pasajeros relativamente pasivas y uniformes. De esta manera, el acento, antes puesto sobre el poder de transito (la velocidad, la capacidad, la amplitud), se desplaza en parte sobre los aspectos "periféricos" o "inmateriales", hasta ahora en un segundo nivel: las interfaces y la accesibilidad, los lugares y la parte "inmóvil" de la movilidad, la facilidad de uso, la relación y los servicios (Pp., 14 -15). Por su parte, Miralles (2001) concluye: "si hasta hace dos décadas hablábamos de tránsito, hace una, de política de transporte y ahora, de movilidad, no es porque nos guste cambiar de palabra, sino porque hemos ido comprendiendo la dinámica de los traslados de las personas" (citado por Avellaneda, 2007, p. 104).

No obstante, si bien hay una inclinación hacia el sujeto, perdura un sesgo materialista en el abordaje del encuentro de éste con su territorio. La satisfacción de necesidades pasa por la llegada a lugares; y la movilidad, la accesibilidad o los itinerarios se explican por la localización, sea de las actividades, de la residencia y las funciones urbanas que las articulan. El desplazamiento territorial y su vinculación con el espacio urbano se lee desde la configuración material del territorio. (Gutiérrez, 2012)

Por otra parte, la movilidad y la oferta de los servicios de transporte raramente han sido estudiadas en sus relaciones con los grupos sociales menos favorecidos, es decir, aquellos que poseen exiguos niveles de ingresos o rentas. Es necesario advertir que en el texto, el término movilidad refiere a la movilidad urbana, es decir, a una movilidad territorial, no así a una movilidad social (cambio de clase social).

En este sentido, la accesibilidad se presenta como uno de los nexos que puede asumir el abordaje de la relación movilidad-pobreza; entendiendo que la accesibilidad a las oportunidades de empleo, a los lugares de residencia y a los espacios donde se ofrecen muchos de los servicios esenciales dependen en gran medida de las posibilidades y circunstancias materiales de los viajes.

Publicado en formato digital: Lic. Hector Daniel Blanco. UNA MIRADA A LA POBLACIÓN DE BAJOS INGRESOS DEL ÁREA METROPOLITANA DE POSADAS DESDE LA MOVILIDAD COTIDIANA. Revista Geográfica Digital. IGUNNE. Facultad de Humanidades. UNNE. Año 12. NN 24. Julio - Diciembre 2015. ISSN 1668-5180 Resistencia, Chaco.

En: http://hum.unne.edu.ar/revistas/geoweb/default.htm 
Avellaneda (2008), sostiene que entre la movilidad y la pobreza, se establecen estrechos vínculos y fuertes relaciones e interdependencias. Sin duda alguna la situación de pobreza condiciona las pautas de movilidad de los sectores populares de la población. Del mismo modo, la posibilidad de integrar la movilidad a la vida cotidiana modula los procesos de integración y cohesión sociales. Así, entre ambas variables se establece una relación biunívoca en la que una y otra interactúan profundamente entre sí (p.17).

De esta manera, el propósito fundamental del artículo es analizar - de manera escueta- los viajes realizados en el Área Metropolitana de Posadas (en adelante AMP), con el énfasis puesto en los grupos sociales de menores recursos económicos, entendiendo, a su vez, que los mismos viajes- son solo una manifestación parcial de la movilidad de un grupo social, donde los problemas de movilidad pueden ser, con frecuencia, agravantes de la pobreza y, por ende, de la exclusión social, puesto que los espacios, tiempos y modalidades de la movilidad están diferenciados en función del nivel de riqueza (Delaunay, 2007, citado por Jouffe, 2011, p. 85). En este contexto, si bien es cierto que existe una correspondiente relación entre el transporte y la movilidad, vale aclarar que dicha reciprocidad no es suficiente para conocer la movilidad. Es así que se intenta proponer una visión analítica de los desplazamientos de la población de bajos ingresos económicos (indigentes y pobres) en el territorio del AMP, desde una dimensión material, es decir, según los viajes efectivamente realizados.

Para el desarrollo del presente escrito, se han considerado indicadores que conciernen a la relación movilidad-ingreso y que conforman los datos secundarios de las Encuestas de Origen Destino (en adelante EOD), aplicada al AMP en el año 2010. Las encuestas de movilidad cotidiana constituyen una útil herramienta a los fines de recolectar información referida en particular a cuestiones como el medio de transporte, el motivo del viaje, la hora de salida y de llegada, el origen y el destino, entre otras. Dicha información, desde luego tiene un gran valor, en tanto, cuantificación y representación de patrones de desplazamiento de la población de un área de estudio. No obstante, es menester reconocer que la comprensión de la movilidad de manera integral no concluye en los viajes materializados, puesto que también incluye un universo subjetivo que requiere otras herramientas y definiciones metodologías adicionales para su correcto tratamiento. Asimismo, las EOD partir de sus alcances y limitaciones pueden convertirse en un instrumento de reflexión para el estudio de la movilidad.

\section{EL ÁREA Y LA POBLACIÓN DE ESTUDIO}

Tal como se ha apuntado, la movilidad se explica y se comprende desde dimensiones materiales y subjetivas, pero también es cierto que la misma es llevada en un espacio material, en este caso el AMP aglomerado urbano que en la EOD (2010) abarcó a la ciudad de Posadas y a las localidades de Garupá y Candelaria (Figura 1).

Si bien, las relaciones de movilidad y pobreza no son exclusivas de ninguna ciudad o región del planeta, se ha optado por analizar el caso particular del AMP, donde el 27,5\% de los hogares se encuentran debajo de la línea de pobreza y de indigencia según la EOD (2010), razón por la cual, se puede considerar como una de las áreas metropolitanas intermedias de la República Argentina, donde la participación de estos grupos socioeconómicos de bajos ingresos es bastante significativa.

En las Áreas Metropolitanas de Tucumán y Mendoza, se encuentran por debajo de la línea de pobreza y de indigencia el $11,3 \%$ y $16,1 \%$ de los hogares respectivamente, según las EOD de los años 2011 y 2010.

Publicado en formato digital: Lic. Hector Daniel Blanco. UNA MIRADA A LA POBLACIÓN DE BAJOS INGRESOS DEL ÁREA METROPOLITANA DE POSADAS DESDE LA MOVILIDAD COTIDIANA. REVISTa Geográfica Digital. IGUNNE. Facultad de Humanidades. UNNE. Año 12. N No 24. Julio - Diciembre 2015. ISSN 1668-5180 Resistencia, Chaco.

En: http://hum.unne.edu.ar/revistas/geoweb/default.htm 
Figura 1: Área Metropolitana de Posadas.

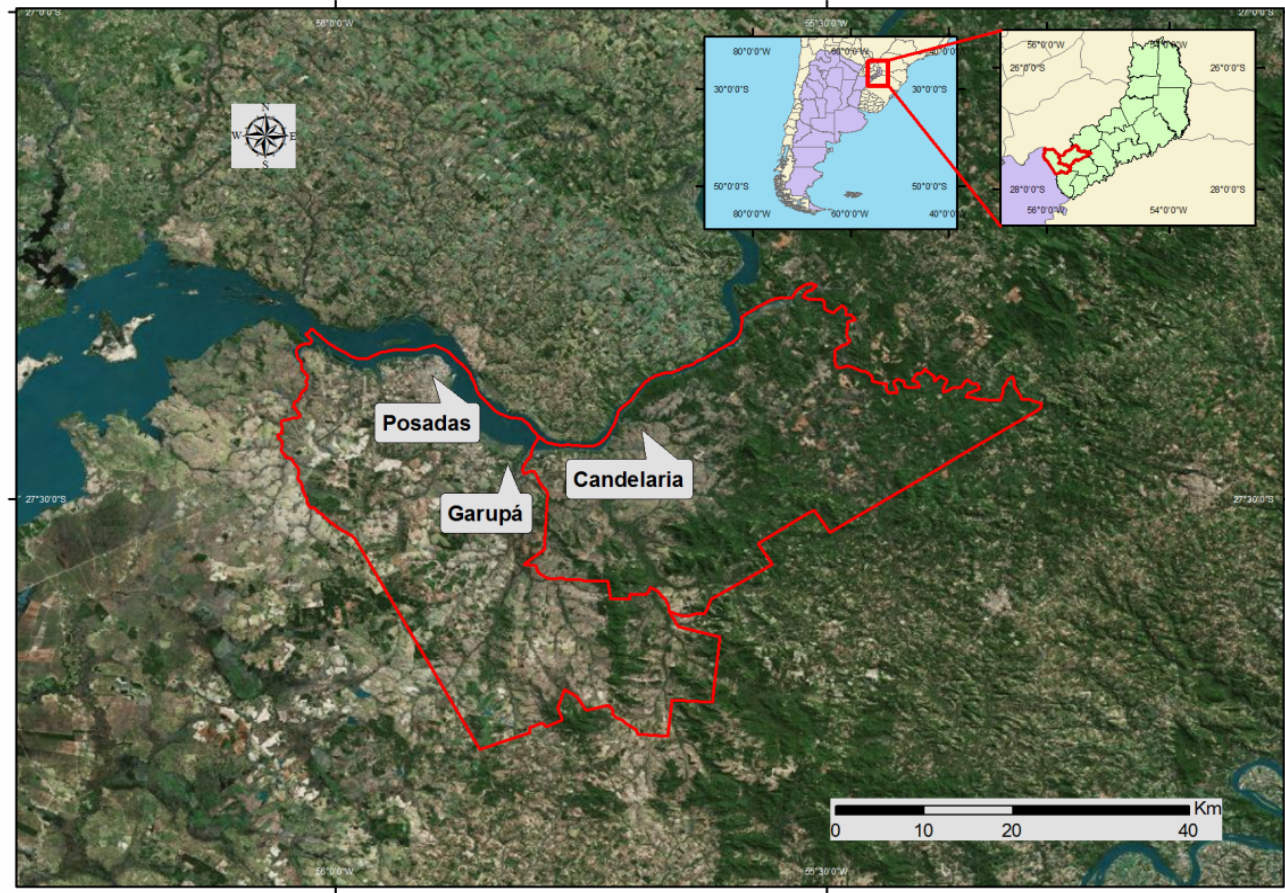

Fuente: elaboración propia.

Entonces, de acuerdo con lo señalado anteriormente, es de orden realizar una breve caracterización del contexto sociodemográfico y económico del ámbito de estudio, que incluye, -por criterios que tienen que ver con la existencia de un proceso de vinculación funcional con respecto a los alcances y cobertura del sistema de transporte-, a las jurisdicciones de Posadas, Candelaria y Garupá, tal como se ha expuesto anteriormente.

El $14 \%$ de la población en estudio posee sus residencias en las localidades de Candelaria y Garupá, aunque estos lugares poseen un promedio de personas por hogar más alto que la ciudad de Posadas, capital provincial donde se localiza el $86 \%$ la población restante, actuando por tal razón como núcleo del área metropolitana, dado que constituye un centro polifuncional con servicios de alta complejidad y especialización (EOD, 2010, Posadas), siendo uno de los primeros factores influyentes en las decisiones de movilidad de las personas y en el uso de dicho espacio urbano. Al respecto, Vansconcellos (2010) en su obra titulada "Análisis de la movilidad urbana. Espacio, medio ambiente y equidad", expresa lo siguiente:

Las distintas maneras a través de las cuales el desarrollo urbano ocurrió originalmente en los países en desarrollo ha traído consecuencias de gran impacto en las condiciones de movilidad de las personas.

-La más significativa, sin duda, es la ubicación de las personas en relación con sus necesidades de trabajo, educación y tiempo destinado al ocio. En la mayor parte de las grandes ciudades la población de bajos ingresos ocupa regiones periféricas, donde el valor de la tierra o la posibilidad de adquisición de áreas libres y sin costos ha permitido la construcción de un hogar.

En dichas áreas, la oferta de servicios públicos-como escuelas, centros de salud y transporte colectivo- es precaria y a ello se suma una limitada oferta de oportunidades de trabajo, tanto en cantidad como en variedad. (p. 26)

En este sentido, la EOD (2010) no ofrece información de manera desagregada sobre la localización de las diferentes actividades (educativas, recreativas, laborales, comerciales, etc.) y de la población en general, condiciones que si bien son objetivas en la movilidad, influyen en la igualdad de oportunidades que poseen los habitantes desde la perspectiva territorial para ser participes de la vida en la ciudad, participación que solo puede ser concebida a partir de las garantías de acceso de todas

Publicado en formato digital: Lic. Hector Daniel Blanco. UNA MIRADA A LA POBLACIÓN DE BAJOS INGRESOS DEL ÁREA METROPOLITANA DE POSADAS DESDE LA MOVILIDAD COTIDIANA. Revista Geográfica Digital. IGUNNE. Facultad de Humanidades. UNNE. Año 12. № 24. Julio - Diciembre 2015. ISSN 1668-5180 Resistencia, Chaco.

En: http://hum.unne.edu.ar/revistas/geoweb/default.htm 
las personas a los diferentes sectores de la urbe, permitiendo de este modo la inclusión, la integración y la cohesión social.

No obstante, la concreción de tales ejercicios y metas de ciudadanía plena de la sociedad, dependerá no solo de la estructura urbana, de la organización territorial y del servicio de transporte del lugar donde se realicen sus actividades cotidianas sino también de las características socioeconómicas de cada individuo (Avellaneda, 2008), y de factores actitudinales, de valores, experienciales y de percepción que tienen que ver más bien con aspectos conductuales de las personas, así como de sus criterios de consumo (Hernández et al., 2011).

Para esta área de estudio, la EOD estableció una población para el año 2010 de 334.059 habitantes y un total de 98.630 hogares, los cuales difieren con los datos preliminares del Censo Nacional de Población, Hogares y Vivienda 2010. De dicha población, han sido encuestados un total de 1731 hogares, 5940 personas y relevados 10241 viajes.

Tal diferencia se debe a que la EOD 2010, considera la población de cada una de las tres jurisdicciones, es decir, Posadas, Candelaria y Garupá, mientras que el Censo Nacional de Población, Hogares y Vivienda 2010 no ha tenido en cuenta a la localidad de Garupá.

En la composición demográfica del Área Metropolitana de Posadas, en lo que refiere a la población según sexo, se puede observar una mayor la proporción de mujeres (53,3 \%) que de hombres (46,3\%), mientras que en la estructura etaria es mayor la participación de jóvenes de entre 15 y 19 años.

Vale recordar que los datos referidos a la población total y sus correspondientes disgregaciones derivan de los resultados obtenidos de la EOD 2010. Consecuentemente, y de acuerdo con el criterio de considerar a la población mayor de 4 años de edad, la misma está constituida por 310. 239 personas, de las cuales un 68,30\% realizo viajes el día anterior a la Encuesta, es decir, 211.783 individuos. Este grupo tiene un promedio de 29 años aproximadamente.

La edad impacta directamente en la movilidad relacionada con las tareas que son atribuidas, aceptadas o esperadas por parte de cierto grupo de personas, de acuerdo con las condiciones sociales. Como la movilidad está primordialmente relacionada con el trabajo, las personas en fase "productiva" -entre los 20 y los 50 años- generalmente se desplazan más. Considerando que la escuela es la segunda mayor causa de desplazamientos en la mayoría de los lugares, los niños y los jóvenes también son considerados muy "móviles". Por su parte, los niños en etapa pre escolar y los jubilados aparecen en el grupo de los menos móviles Vasconcellos (2010, p. 33).

En cuanto al género, Peters (1998, p.12), enfatiza "las principales diferencias en las necesidades básicas de movilidad de hombres y mujeres están relacionadas con la división del trabajo, influenciada por el género dentro de la familia y la comunidad". En la mayoría de los casos, las actividades externas al hogar son atribuidas principalmente a los hombres adultos, mientras las actividades dentro de la casa son atribuidas a las mujeres adultas. La primera consecuencia de esta división es que en la mayoría de las sociedades las mujeres son menos móviles que los hombres. El papel de las mujeres tiene especial importancia en la comprensión de los patrones diarios de desplazamiento en los hogares de los países en desarrollo (ibíd, p. 34).

Las características de la movilidad de las mujeres, como grupo urbano, derivan del hecho de que no solo dependen de su nivel de renta y de la edad, sino que ésta se origina, fundamentalmente, a partir del papel que tienen en la sociedad. Las mujeres, por tradición, han ocupado lugares preferentes en la esfera privada y familiar; en este sentido, sus desplazamientos se dan en un ámbito más cotidiano y más próximo al lugar de residencia que aquellos que, en general, realizan los hombres (Pickup, 1989, citado por Miralles, 2002, p. 30)

Ahora bien, resulta necesario, a los fines del presente escrito, remitirse a la situación de los grupos sociales (indigentes y pobres) que en suma representan al 27, $5 \%$ de la población, los cuales dado sus ingresos no pueden cubrir dichas necesidades de bienes y servicios no alimentarios, donde se incluye claramente al transporte como vector de movilidad y del acceso a otros servicios como la salud y la educación. Aun más, según Amar (2011), la movilidad deviene de un cuasiderecho social, como la salud o la educación, o un bien público como el agua o la electricidad, del que nadie debería ser privado; del mismo modo, Manuel Herce (2009), entiende que el derecho a la movilidad es el derecho de todo el mundo a ir a todas partes.

Publicado en formato digital: Lic. Hector Daniel Blanco. UNA MIRADA A LA POBLACIÓN DE BAJOS INGRESOS DEL ÁREA METROPOLITANA DE POSADAS DESDE LA MOVILIDAD COTIDIANA. REVISTa Geográfica Digital. IGUNNE. Facultad de Humanidades. UNNE. Año 12. № 24. Julio - Diciembre 2015. ISSN 1668-5180 Resistencia, Chaco.

En: http://hum.unne.edu.ar/revistas/geoweb/default.htm 


\section{DESARROLLO}

\section{LOS BAJOS INGRESOS Y LA MOVILIDAD REALIZADA EN EL AMP}

En lo que respecta a ingresos económicos, la encuesta muestra que en el 2010, el 4,7 \% de los hogares se encuentran en situación de indigencia (con ingresos que no superan la canasta básica de alimentos de $\$ 596,14$ ), un $22,8 \%$ de hogares pobres, es decir, cuyos ingresos no son superiores al valor de la canasta básica total $(\$ 1.314,16)$, y un $69,2 \%$ de hogares no pobres, aquellos que tienen la capacidad de satisfacer sus necesidades esenciales utilizando una canasta básica de alimentos, además de la inclusión de bienes y servicios no alimentarios (vestimentas, transporte, salud, educación, etc.). La pobreza urbana, está vista aquí no como un objeto aislado de estudio sino con relación a la movilidad.

En contextos socioeconómicos como el descrito anteriormente, se puede inferir que el nivel de renta es, sin duda alguna, una de las principales variables que condicionan o modulan la forma de desplazarse de los ciudadanos. Lamentablemente la EOD (2010), para el caso del AMP, no brinda información para examinar el gasto destinado por este grupo social al transporte. Con frecuencia las personas que se encuentran en situación de pobreza se ven obligadas a vivir en las áreas periféricas de la ciudad, a menudo lejos de las oportunidades de empleo y de los servicios básicos. Ello les obliga a realizar importantes desplazamientos para realizar sus actividades cotidianas. Las posibilidades de realizar estos desplazamientos dependerán, fundamentalmente, de la capacidad de sufragar el coste de dichos desplazamientos. Por ello, los costes de transporte tienen impactos significativos sobre las posibilidades de desplazamiento de las familias de rentas bajas. Los miembros de hogares pobres suelen destinar importantes porcentajes de sus presupuestos familiares a los desplazamientos (Booth, et. al, 2000) siendo uno de los principales ítems de los gastos de dichas familias (Fundación Ciudad, 2000; Kaltheier, 2002; Ávila, 2003; Itrans, 2004 citado por Avellaneda, 2008, p. 17)

En la EOD 2010 del AMP, la variable de ingresos fue construida a partir de los datos declarados por los encuestados sobre el ingreso del hogar. Luego se establecieron quintiles de ingresos, donde se dividió la población en cinco partes iguales según su ingreso per-cápita. Esta variable en particular fue cruzada con otras, obteniéndose algunos de los resultados que se presentan y analizan a continuación, poniendo siempre mayor énfasis en los hogares que pertenecen a los quintiles de ingresos más bajos.

\section{LOS MEDIOS Y LOS MODOS}

Se observa entonces, y como era de esperar, que la mayor cantidad de hogares con medios motorizados está asociada a los quintiles de ingresos más altos, mientras que la bicicleta como medio no motorizado es más común en los grupos de ingresos más bajos (Gráfico 1). No obstante, es llamativo que más de la mitad de los hogares no cuenten con ningún vehículo motorizado (Tabla 1), en un contexto donde según los resultados de las encuestas un $67 \%$ de los viajes que se realizan en el AMP se efectúan en transporte motorizado.

Tabla 1: Tenencia de vehículos y bicicletas por hogar

\begin{tabular}{cccc}
\hline Características de los hogares & AMP & Posadas & AMsP* \\
\hline$N^{\circ}$ de hogares & 98.630 & 84.827 & 13.803 \\
Personas por hogar (promedio) & 3,39 & 3,34 & 3,66 \\
Hogares con un auto (\%) & 27,22 & 27,84 & 23,36 \\
Hogares con más de un auto (\%) & 3,57 & 3,65 & 3,08 \\
Hogares con moto/ciclomotor (\%) & 17,64 & 17,76 & 16,88 \\
Hogares con bicicleta & 35,98 & 34,58 & 44,58 \\
Hogares sin vehículo motorizado (sin & 57,08 & 56,41 & 61,20 \\
auto ni moto) & & & \\
\hline
\end{tabular}

Fuente: EOD 2010 Posadas (PTUMA) **

(*) Área Metropolitana sin Posadas

$\left.{ }^{* *}\right)$ Proyecto de transporte urbano para áreas metropolitanas

Publicado en formato digital: Lic. Hector Daniel Blanco. UNA MIRADA A LA POBLACIÓN DE BAJOS INGRESOS DEL ÁREA METROPOLITANA DE POSADAS DESDE LA MOVILIDAD COTIDIANA. Revista Geográfica Digital. IGUNNE. Facultad de Humanidades. UNNE. Año 12. NN 24. Julio - Diciembre 2015. ISSN 1668-5180 Resistencia, Chaco.

En: http://hum.unne.edu.ar/revistas/geoweb/default.htm 
El caso particular del AMP, se inscribe en el conjunto de ciudades de América Latina, donde tal como indica Vasconcellos (2005, citado por Avellaneda, 2008), debido a la importante distancia que separa las distintas funciones urbanas, la accesibilidad viene determinada principalmente por las posibilidades de uso de medios de transporte motorizados. Es así que el uso de diversas formas de transporte está fuertemente influenciado por el nivel de ingresos. Personas con bajos ingresos desempeñan mucho más el papel de peatones, ciclistas y usuarios de transporte público; personas con ingresos más altos suelen desempeñarse como motoristas o pasajeros de automóviles (p. 17). Asimismo, también puede afirmarse que a mayor nivel de renta mayor utilización de medios de transporte motorizados individuales, fundamentalmente automóviles (Kauffman, 2000, ibíd, p. 18). De esta manera, el ingreso tiene una fuerte influencia en la elección de los modos de desplazamiento (Vasconcellos 2010), tal como puede notarse en los Gráficos 1 y 2.

Grafico 1: Porcentaje de hogares con auto, moto y bicicleta según quintil

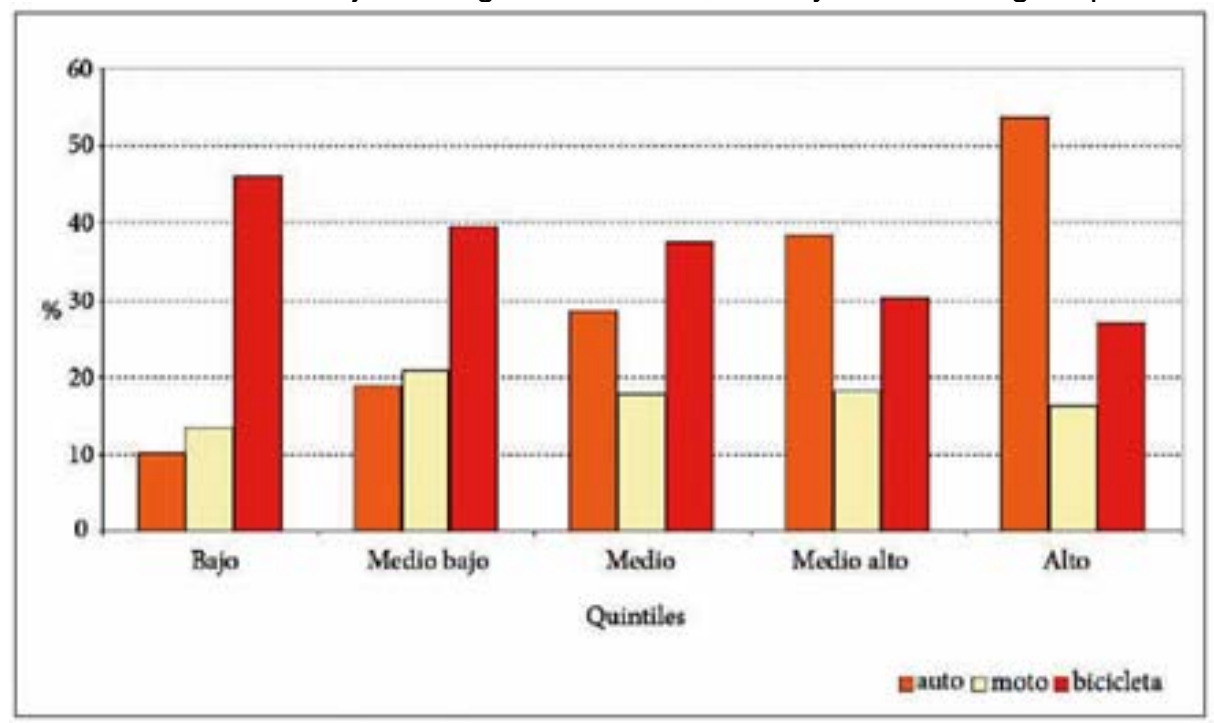

Fuente: EOD 2010 Posadas (PTUMA)

Al incorporar al análisis la información sobre los quintiles de ingreso de los hogares, resulta llamativo observar que el uso del colectivo es parejo en todos los grupos (disminuyendo lentamente hacia el quintil más alto), a diferencia del uso del automóvil como conductor/acompañante que crece a medida que aumenta el nivel de ingreso en los hogares. Con referencia a esto, Orfeuil (2010), sostiene que la capacidad de movilidad diversificada siempre ha estado vinculada, y de manera significativa a los recursos del hogar, y no hay duda de que la propagación del automóvil a los sectores más pobres de la población ha contribuido en cierta democratización de los usos del espacio. Sin embargo, esta situación no hace sino reforzar la brecha diferencial de aquellos que no están motorizados (más de la mitad de los hogares del AMP), o que no tienen la licencia, que para este caso, según la EOD (2010), corresponde al 67 \% de la población mayor de 17 años.

La posesión de un vehículo motorizado es un elemento clave del nivel de accesibilidad, a la vez no poseerlo se convierte en un factor de exclusión social (Rodriguez, et. al., 2006 citado por Lizarraga, 2012, p. 102). Los bajos ingresos obligan generalmente a los pobres a compensar sus limitadas opciones de movilidad con tiempos de viaje más prolongados y con un mayor uso de las modalidades de transporte no motorizado, como caminar y andar en bicicleta. En general, esas modalidades han recibido menos atención a nivel normativo, así como inversiones mínimas en los ámbitos de políticas y desarrollo del transporte urbano (Grieco, 2013)

De manera contraria se observa que los viajes a pie disminuyen a medida que aumenta el quintil de ingreso, tal como se puede observar en el Grafico 2.

Publicado en formato digital: Lic. Hector Daniel Blanco. UNA MIRADA A LA POBLACIÓN DE BAJOS INGRESOS DEL ÁREA METROPOLITANA DE POSADAS DESDE LA MOVILIDAD COTIDIANA. Revista Geográfica Digital. IGUNNE. Facultad de Humanidades. UNNE. Año 12. N No 24. Julio - Diciembre 2015. ISSN 1668-5180 Resistencia, Chaco.

En: http://hum.unne.edu.ar/revistas/geoweb/default.htm 
Grafico 2: distribución modal de viajes en el AMP para cada quintil



Fuente: EOD 2010 Posadas (PTUMA)

En la Tabla 2, a partir de la relación entre la tasa de generación de viajes y la situación de bienestar, se manifiesta que los sectores considerados no pobres realizan mas viajes que los más pobres, lo cual también aparece asociado a la tenencia de auto en el hogar (Grafico 1) y consecuentemente la mayor tasa de generación de viajes en comparación con aquellos que no lo poseen. Esta situación según Vasconcellos (2010), puede ser considerada como un fenómeno universal, independientemente de las condiciones geográficas o sociales. Este autor señala que dentro de cualquier sociedad, la movilidad aumenta cuando el ingreso aumenta. Esto significa que en una determinada ciudad las personas con mayores ingresos se desplazan con más frecuencia que aquellas de menores ingresos: la relación entre los extremos de la movilidad puede alcanzar de tres a cuatro viajes por persona al día.

Tabla 2: generación de viajes según situación de bienestar

\begin{tabular}{lccccc}
\hline Bienestar & $\begin{array}{c}\text { Tasa de } \\
\text { generación de } \\
\text { viajes por } \\
\text { persona que } \\
\text { viaja }\end{array}$ & $\begin{array}{c}\text { Tasa de } \\
\text { generación de } \\
\text { viajes por } \\
\text { persona }\end{array}$ & $\begin{array}{c}\text { Tasa de } \\
\text { generación de } \\
\text { viajes por } \\
\text { hogar }\end{array}$ & $\begin{array}{c}\text { Tasa de } \\
\text { generación de } \\
\text { viajes por } \\
\text { hogar con } \\
\text { auto }\end{array}$ & $\begin{array}{c}\text { Tasa de } \\
\text { generación de } \\
\text { viajes por } \\
\text { hogar sin auto }\end{array}$ \\
\hline No pobre & 2,72 & 1,92 & 6,42 & 7,04 & 6,03 \\
Pobre & 2,58 & 1,56 & 4,30 & 6,65 & 4,02 \\
Indigente & 2,36 & 1,45 & 3,39 & 6,82 & 3,28 \\
Total & 2,68 & 1,83 & 5,76 & 6,94 & 5,23 \\
\hline
\end{tabular}

$\left(^{*}\right)$ El total de personas y la cantidad de viaje están calculados sobre la población mayor de 4 años.

Fuente: EOD 2010 Posadas (PTUMA)

\section{LOS MOTIVOS DE VIAJES}

En el grafico 3, referido a los motivos de los viajes (necesidad de cada traslado), resulta evidente que los usuarios pobres viajan proporcionalmente más que los otros grupos poblacionales, por motivos "social y familia". Asimismo, llama la atención la proporción de viajes por compras que realizan estos usuarios, lo cual según la Encuesta puede deberse a la resolución cotidiana de las necesidades de aprovisionamiento vinculada con la frecuencia de los ingresos (generalmente

Publicado en formato digital: Lic. Hector Daniel Blanco. UNA MIRADA A LA POBLACIÓN DE BAJOS INGRESOS DEL ÁREA METROPOLITANA DE POSADAS DESDE LA MOVILIDAD COTIDIANA. Revista Geográfica Digital. IGUNNE. Facultad de Humanidades. UNNE. Año 12. N No 24. Julio - Diciembre 2015. ISSN 1668-5180 Resistencia, Chaco.

En: http://hum.unne.edu.ar/revistas/geoweb/default.htm 
ingresos diarios o semanales). Es decir, esta situación podría vincularse con las estrategias de supervivencia que suelen desarrollar estos sectores que incluyen la participación en organizaciones y movimientos sociales, etc.; lo cual contradice a planteamientos como los de Avellaneda (2008), quien afirma que "las dificultades de desplazamiento de las personas en situación de pobreza harán que la mayor parte de los desplazamientos realizados por este grupo social sean por motivos laborales o educativos (Allport, 2000; Ávila, 2003) minimizando los viajes para satisfacer otras necesidades cotidianas tales como ocio, salud o visitas a amigos y familiares. Si bien los sectores populares tienen los mismos motivos que el resto de estratos socioeconómicos para desplazarse, la proporción de estos últimos motivos de desplazamientos solo adquirirá proporciones relevantes entre los grupos sociales de rentas medias y altas" (p. 18)

Respecto a las necesidades de traslado, "los movimientos de las personas en la ciudad actual no pueden explicarse únicamente a partir de la teoría de las necesidades, puesto que, aunque éstas existen (a menudo, la realización de una actividad depende de la posibilidad de desplazarse), eso no permite explicar completamente los desplazamientos que revelan las estrategias de consumo de bienes culturales y sociales" (Haumont, 1993 citado por Miralles, 2002, Pp. 34-35).

Gráfico 3: Distribución de motivos de viajes de personas según situación de bienestar

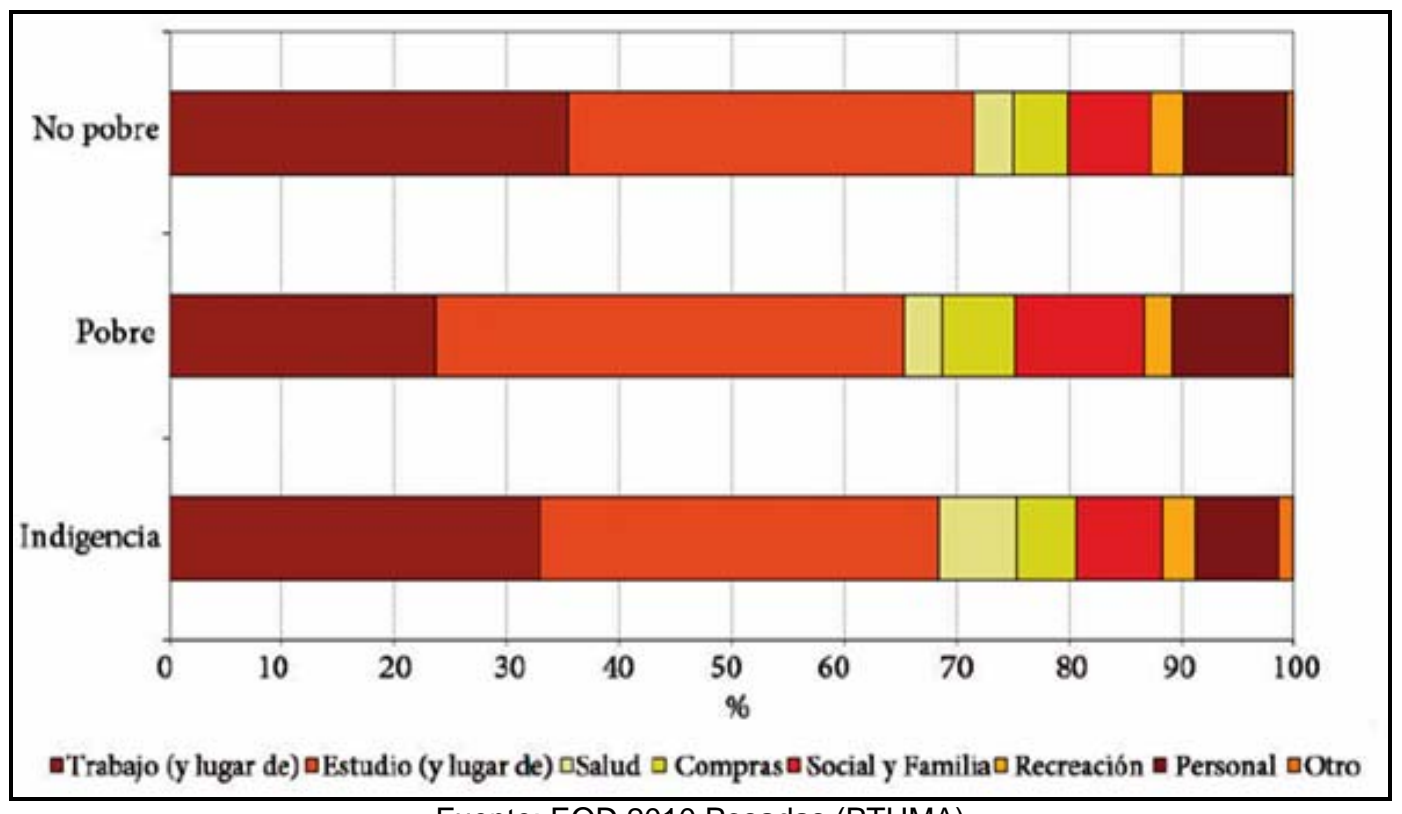

Fuente: EOD 2010 Posadas (PTUMA)

\section{A MODO DE CIERRE}

Lejos de llegar analizar y comprender la movilidad de una manera acadaba, la EOD (2010, Posadas), como herramienta de generación de información, ha permitido, mediante la consideración de la relación movilidad-ingreso, proporcionar un breve panorama de la movilidad materializada en un área que posee un considerable porcentaje de hogares que se encuentran debajo de la línea de pobreza y de indigencia. No obstante, es más que obvio que este tipo de herramientas solo cubre parte de la demanda real de transporte. Esto porque refiere a los viajes efectivamente realizados, solapando otras dimensiones de la movilidad, cuestión no menor, más aun al tratarse de un grupo social que a priori posee como principales problemas, la inequidad en el acceso físico y económico al espacio. Dificultades materiales, que naturalmente nutren a la movilidad y a los mismos viajes realizados, de todo un conjunto de particularidades subjetivas, que para su correcto tratamiento, tal como se ha expuesto en la sección introductoria de este escrito, requieren de otras herramientas y

Publicado en formato digital: Lic. Hector Daniel Blanco. UNA MIRADA A LA POBLACIÓN DE BAJOS INGRESOS DEL ÁREA METROPOLITANA DE POSADAS DESDE LA MOVILIDAD COTIDIANA. Revista Geográfica Digital. IGUNNE. Facultad de Humanidades. UNNE. Año 12. № 24. Julio - Diciembre 2015. ISSN 1668-5180 Resistencia, Chaco.

En: http://hum.unne.edu.ar/revistas/geoweb/default.htm 
definiciones metodologías adicionales en pos de lograr un estudio de mayor profundidad y que permitan distintas miradas complementarias.

Por último interesa rescatar un párrafo del artículo titulado ¿Qué es la movilidad? Elementos para (re)construir las definiciones básicas del campo del transporte; en el que se lee: "Aún queda mucho por indagar respecto a la comprensión de prácticas de viaje. La práctica de viaje varía conforme a una multiplicidad de factores en combinación, entre ellas la edad, el nivel socioeconómico, el género, las aptitudes físicas, cuyo peso relativo difiere y no se conoce con exactitud. Esta conjugación varía entre grupos sociales histórica y territorialmente situados, pero tampoco es estática para un mismo grupo social. Puede variar a lo largo del tiempo, y también conforme a diferentes motivos de viaje (trabajo, salud, educación, compras). Finalmente, la práctica de viaje de un grupo social depende de la propia definición del grupo social. Y sobre esto no se ha avanzado mucho metodológicamente. (Gutiérrez, 2012 p. 70)

\section{BIBLIOGRAFÍA}

- Amar, G. (2011). Homo mobilis: la nueva era de la movilidad. 1 ed. La Crujía. Buenos Aires.

- Avellaneda, P. G. (2008). Movilidad cotidiana, pobreza y exclusión social en la ciudad de Lima. Departamento de Geografía. Universidad Autónoma de Barcelona. Anales de Geografía. vol. 28, núm. 2. Pp. 9-35

- Avellaneda, P. G. (2007). Movilidad, pobreza y exclusión social. Un estudio de caso en la ciudad de Lima. Tesis doctoral. Universitat Autónoma de Barcelona. Departamento de Geografía. Barcelona: Universitat Autònoma de Barcelona.

- Encuesta Origen-Destino 2011. (2012) Movilidad en el Área Metropolitana de Tucumán. $1^{\mathrm{a}}$ ed. Buenos Aires: Proyecto de Transporte Urbano de Buenos Aires.

- Encuesta Origen-Destino 2010. (2012) Movilidad en el Área Metropolitana de Posadas. $1^{\mathrm{a}}$ ed. Buenos Aires: Proyecto de Transporte Urbano de Buenos Aires.

- Encuesta Origen-Destino 2010. (2012) Movilidad en el Área Metropolitana de Mendoza. $1^{\mathrm{a}}$ ed. Buenos Aires: Proyecto de Transporte Urbano de Buenos Aires.

- Grieco, M. (2013). El transporte, la pobreza y el avance hacia sociedades con bajas emisiones de carbono. Boletín FAL. Edición $N^{\circ} 318$, número 2. En:

http://www.cepal.org/Transporte/noticias/bolfall/ FAL-318-WEB.pdf

- Gutiérrez, A. y Minuto D. (2006) Una aproximación metodológica al estudio de lugares con movilidad vulnerable. En: XIV Congreso Latinoamericana de Transporte Público y Urbano. [Actas del XIV CLATPU]. Rio de Janeiro.

- Gutiérrez, A. (2009). "Movilidad o inmovilidad: ¿Qué es la movilidad? Aprendiendo a delimitar los deseos". En: XV Congreso Latinoamericana de Transporte Público y Urbano. [Actas del XV CLATPU]. Buenos Aires.

- Gutiérrez, A. (2012). ¿Qué es la movilidad? Elementos para (re) construir las definiciones básicas del campo del transporte. En: Bitácora 21, № 74. Bogotá: Universidad Nacional de Colombia. Pág. 61-74.

- Herce, M. (2009) Sobre la movilidad en la ciudad. Ed. Revierte. Barcelona.

- Hernández, D. y Witter, R. (2011). Entre la ingeniería y la antropología: hacia un sistema de indicadores integrado sobre transporte público y movilidad. Revista Transporte y Territorio № 4, Universidad de Buenos Aires,. pp. 29-46. En: www.rtt.filo.uba.ar/RTT00403029.pdf

- Jouffe, Y. (2011). Las clases socio-territoriales entre movilidad metropolitana y repliegue barrial. ¿Tienen los pobladores pobres una movilidad urbana de clase?. Revista Transporte y Territorio $\mathrm{N}^{\mathrm{O}}$ 4, Universidad de Buenos Aires, pp. 84-117. www.rtt.filo.uba.ar/RTT00406073.pdf

Publicado en formato digital: Lic. Hector Daniel Blanco. UNA MIRADA A LA POBLACIÓN DE BAJOS INGRESOS DEL ÁREA METROPOLITANA DE POSADAS DESDE LA MOVILIDAD COTIDIANA. Revista Geográfica Digital. IGUNNE. Facultad de Humanidades. UNNE. Año 12. № 24. Julio - Diciembre 2015. ISSN 1668-5180 Resistencia, Chaco.

En: http://hum.unne.edu.ar/revistas/geoweb/default.htm 
Revista Geográfica Digital. IGUNNE. Facultad de Humanidades. UNNE. Año 12. № 24.

Julio - Diciembre 2015. ISSN 1668-5180 Resistencia, Chaco

- Lizarraga, C. (2012). Expansión metropolitana y movilidad: el caso de Caracas. En: Revista EURE, No 113. Santiago de Chile: Pontifica Universidad Católica de Chile.

- Miralles-Gauschs, C. (2002) Ciudad y transporte. El binomio imperfecto. España.

- Orfeuil, J. P. (2010). "Movilidad, nueva cuestión social? ". En: http://sociologies.revues.org/3321

- Vasconcellos A. E. (2010) Análisis de la movilidad urbana. Espacio, medio ambiente y equidad. Bogotá. Colombia. En:

http://omu.caf.com/media/14683/an\%C3\%A1lisis_movilidad_urbana.pdf 